\title{
Die Sprachen von und das Sprechen mit den Tieren in ihren Biographien
}

\begin{abstract}
Spricht die Biographie eine exklusiv menschliche Sprache? Und ist der Mensch die einzig biographiewürdige Spezies? Hält man sich die Tierbiographien vor Augen, die seit dem späten 18. Jahrhundert im deutschsprachigen Raum populär werden, muss zumindest die zweite Frage entschieden verneint werden. Der Beitrag widmet sich diesen Tierbiographien aus einer systematisch und komparatistisch ausgerichteten sowie ästhetisch und funktional interessierten Perspektive. Ein erster Zugriff erfolgt über die Unterscheidung zwischen naturgeschichtlich orientierten ,zoologischen Kollektivbiographien', die das Leben ganzer Arten zur Anschauung bringen, und ,literarischen Zoographien', die das Leben eines bestimmten Tierindividuums in den Blick nehmen wollen. In einem zweiten Schritt folgt eine diachrone Rückschau auf exemplarische Texte des 19. Jahrhunderts und eine Analyse von zwei literarischen Zoographien des 20. und 21. Jahrhunderts - Joaquin Verdaguers Lebensgeschichte eines kleinen Vogels (1966) und Vicki Myrons Dewey (2008). Gefragt wird insbesondere nach dem Verhältnis zwischen tierlicher Biographie und menschlicher Autobiographie, der Verschränkung von Assimilationismus und Anthropomorphisierung sowie den maßgeblichen ästhetischen Verfahren und Funktionen des Sprechens für und über Tierindividuen. Literarischen Zoographien unterliegt ein assimilationistisches Plädoyer u. a. zugunsten einer Sprach- und Kommunikationsfähigkeit der Tiere. Abschließend skizziert der Artikel die Implikationen und Effekte der Sprachen von und des Sprechens mit den Tieren in ihren Biographien. Weder ist dieses zwischen den Polen von Exemplarizität und Exzeptionalität changierende Erzählen bedingungs- und absichtslos, noch lässt sich diesen Tierbiographien ein kritisches (selbst-)reflexives Moment absprechen, das die sprachlichen und epistemologischen Grenzen des Menschen berührt.
\end{abstract}

Keywords: Biographie; Tiere; Naturgeschichte; literarische Zoographie; Assimilationismus; Anthropomorphismus; Prosopopöie; epistemischer Anthropozentrismus 


\section{Biographien der Tiere}

Texte, die das Sprechen über Tiere biographisch ästhetisieren, tauchen in der Blütezeit der Biographie im späten 18. und dann insbesondere im 19. Jahrhundert in zwei Formaten auf. Zum einen handelt es sich hierbei um solche Schriften, in denen die Lebensskizze eines bestimmten Tierindividuums entworfen, verschiedene anekdotenhaft-biographemartige Lebensepisoden dargelegt oder der Charakter besonderer, zum Teil ,berühmter` Tierindividuen schriftlich fixiert wird. Bei diesen Texten, die mal von fiktiven, mal von historisch referentialisierbaren

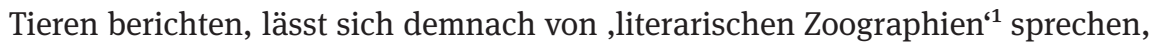
die aus dem Leben eines bestimmten Tierindividuums erzählen, gleichzeitig aber auch Aussagen über die Spezies machen, der das entsprechende Individuum angehört. Literarische Zoographien schreiben somit immer auch an einem spezifischen Artenwissen mit. Zum anderen findet man zoologische Texte, die sich mit den Lebensformen und Charaktereigenschaften bestimmter Tierarten beschäftigen und somit Biographien ganzer Spezies liefern. Verstanden als „Darstellung des Lebens eines Individuums, [...] die tendenziell an Vollständigkeit orientiert ist“ (Schnicke 2009, 2), teilt sich die Biographie ihren Anspruch einer sowohl totalen als auch wahrhaften Lebensbeschreibung mit der Naturgeschichte, der es, mit dem kanonischen Naturforscher des 18. Jahrhunderts, Georges-Louis Leclerc de Buffon, gesprochen, um ,eine genaue Beschreibung und getreue Geschichte von jeder Sache“ (Buffon 1771, 45) und somit auch jeder Spezies geht. Die Parallele zwischen Naturgeschichte und Biographie wurde ab dem späten 18. Jahrhundert noch dadurch verstärkt, dass man naturgeschichtliche Abhandlungen nun auch als ,Lebensgeschichten“ der Tiere bezeichnete. ${ }^{2}$ Aussehen, geographische Verteilung, Lebensraum, Eigenschaften und Verhalten, Dispositionen und Verhältnisse zu anderen Arten waren konstitutive Bestandteile dieser naturgeschichtlichen Tierbiographien. Texte dieser Art lassen sich demnach als ,zoologische Kollektivbiographien' bezeichnen.

Ein frühes Zeugnis der konzeptuellen und ästhetischen Verschränkung von Biographie und Naturgeschichte stellt die mehrbändige Animal Biography (1802) des englischen Pfarrers William Bingley (1774-1823) dar, die bereits zwischen 1804 und 1810 ins Deutsche übersetzt wurde. Bingley geht es nicht darum, eine systematisch-klassifikatorische Einteilung der Fauna vorzustellen. Ziel der Dar-

1 Diese und ähnliche systematisch-terminologische Annäherungen habe ich an anderer Stelle entwickelt (vgl. Middelhoff 2020, u.a. 44-48.). Der Begriff ,Zoographie‘ wurde im späten 18. und im 19. Jahrhundert häufig synonymisch für ,Naturgeschichte‘ verwendet.

2 Vgl. hierzu Toepfer 2011, 497. 
stellung ist vielmehr eine physikotheologisch orientierte, zoomorphe Gestaltung der Lebensgeschichte der Tierarten, der es weniger um ,wissenschaftliche exakte Beschreibung“, sondern vielmehr um eine spannende Erzählung des Tierlebens sowie „die episodenhafte Veranschaulichung der Lebensbedingungen und die literarisch avancierte Darbietung von charakteristischen Szenen“ (Schmideler 2012, 49) geht. Inhaltlich stehen daher „anecdotes and observations“ von den „habits of life and instincts of the animals“ (Bingley ${ }^{2} 1804, \mathrm{v}$ ) im Vordergrund. Da er all die Beschreibungen ausspart, die nicht unmittelbar die „characters of the animals“ illustrieren, erscheint Bingley der Begriff der Animal Biography durchaus passend:

[S]ince Biography is a term that, by long usage, has become exclusively appropriated to the lives of individuals among mankind, the term Animal Biography may surely, and without impropriety, be considered to express traits of the lives and habits of individual species of the lower orders of the animate creation, as distinct from those of men. This is the precise signification in which it is here used. But doubtless, the compound of $\beta \iota \circ$ and $\gamma \rho \alpha \varphi \omega$ is as applicable to the lives of animals, as to those of men. (Bingley ${ }^{2} 1804$, vi)

Zwar bezieht Bingley den Begriff der Biographie aus einer etymologischen Perspektive in einem prinzipiell demokratisierenden Gestus auf die Beschreibung der Charakter- und Lebenseigenschaften der Tiere, nimmt aber gleichzeitig eine Grenzziehung zwischen dem (Er-)Leben von Menschen („mankind“) und Tieren (,lower orders of the animate creation“) vor. Den Begriff des Individuums appliziert er dabei auf die einzelnen Spezies, nicht auf einzelne Tiere. Ein Bewusstsein dafür, dass Tiere der gleichen Spezies individuelle Charakterzüge tragen könnten, ist bei Bingley (noch) nicht maßgeblich.

Auch in den Biographieen aus der Naturkunde (1851), die der Volksschulpädagoge August Wilhelm Grube (1816-1824) herausgab, ${ }^{3}$ soll einer Privilegierung „systematische[n] Wissen[s]“ (21851, vi) entgegengearbeitet werden. Grube erteilt einer „Anschauung von Individuen“ (21851, vi) in der Naturgeschichte den Vorzug und geht dementsprechend auf die Lebensweisen und Eigenschaften ausgewählter Spezies (u. a. Honigbiene, Schildkröte, Hund, Pferd) ein. Sein didaktisches Darstellungsverfahren, das vordergründig auf die Unterrichtung von Kindern und Jugendlichen ausgerichtet ist, bezeichnet Grube daher als „biographische Methode“, die „nur Einzelnes, Weniges darreicht, aber solches desto ausführlicher, desto mehr mit Liebe behandelt, und eben deshalb den Schüler desto mehr

3 Vgl. Schmideler 2011. 
in das Objekt vertieft“ (21851, vi) und für die Einzigartigkeit der göttlichen Schöpfung sensibilisiert. Auch Grube erzählt von Tierarten, nicht von Tierindividuen.

An der Schnittstelle von Biographie und Zoologie, von zoologischer Kollektivbiographie und literarischer Zoographie steht das Werk Alfred Edmund Brehms (1829-1884). In seinem Illustrirten Thierleben, dem wohl populärsten zoologischen Werk des 19. Jahrhunderts, macht Brehm es sich zur Aufgabe, Tiere nicht als einen „todte[n], ausgetopfte[n], in Weingeist aufbewahrte[n]“ Gegenstand, sondern als „fühlende[ ] und bewegungsfähige[ ]“, als „handelnde[ ] und wirkende[ ] Wesen“ (1864, vii) wahrzunehmen und diese Lebendigkeit sprachlich zu repräsentieren: Leben(dig) erzählen war spätestens mit Brehm zum sine qua non naturkundlicher Programmatik geworden. ${ }^{4}$ Die ,Verlebendigung eines bestimmten Gegenstandes mit sprachlichen Mitteln wurde in diesem Zuge zu einem ästhetischen Anspruch, den sich Literatur und Zoologie teilten.

Brehms Werk markiert gleichzeitig einen Kulminationspunkt assimilationistischer Vorstellungen vom Geist der Tiere. Während Differentialisten „,von einem prinzipiellen Unterschied zwischen Menschen und anderen Tieren“ (Wild 2006, 2) u.a. qua Sprach- und Vernunftfähigkeit ausgehen, nehmen Assimilationisten an, dass eine „Entwicklungskontinuität verschiedener Tierarten, zu denen der Mensch mit gehört“ (Wild 2006, 9), besteht. Als assimilationistischer Darwinist kommt Brehm zu dem Schluss, dass Säugetiere über dem Menschen vergleichbare, quasi-autobiographische Kompetenzen verfügen: ${ }^{5}$ „Das Säugethier besitzt Gedächtniß, Verstand und Gemüth [...]. Das kluge Thier rechnet, bedenkt, erwägt, ehe es handelt [...]. Es überwindet Begierden und Leidenschaften und lernt sich beherrschen [...]. Es erinnert sich der Vergangenheit jahrelang und gedenkt sogar der Zukunft.“ (Brehm 1865, xxvii-xxviii) Gleichzeitig ist Brehm auch davon überzeugt, dass Tiere über ein Selbstausdrucksvermögen verfügen: „Die Bewegung der Thierseele spricht aus dem Auge; dieses ersetzt die fehlende Sprache“ (Brehm 1864, 37). Wer sich mit dem Leben und den Verhaltensweisen der Tiere auskennt, vermag laut Brehm auch, die Gemütszustände und die Absichten der Tiere aus ihrer Körper- und Zeichensprache zu begreifen bzw. diese Zeichen, gleich einem offenen Buch, zu ,lesen'.

Charakterskizzen und Biographeme bestimmter Tierindividuen sind dabei nicht nur in Brehms Illustrirtem Thierleben Teil des anekdotisch-lebendigen Erzählens. 1866 veröffentlicht die Familienzeitschrift Die Gartenlaube Brehms

$4 \mathrm{Zu}$ dieser Ästhetisierung in den Naturwissenschaften vgl. insbesondere Müller-Tamm 2010. 5 Vgl. zu diesen Zuschreibungen im Kontext einer assimilationistischen Tiertheorie, die sich vor dem Hintergrund der Diskussion über die Tierseele bis ins 18. Jahrhundert zurückverfolgen lässt, Ingensiep (1996) sowie Middelhoff (i.E.). 
,literarische Zoographie“ der im Hamburger Zoo lebenden Schimpansin Molli. Der Text geht nicht nur auf Herkunft, Charakter und Ableben der Schimpansin, sondern auch auf die Sprach- und Interaktionsfähigkeit des Tieres ein. Brehm schreibt:

Er [d.i. der Schimpanse] versteht, was gesprochen wird, und wir verstehen auch ihn, weil er zu sprechen weiß - nicht mit Worten allerdings, aber mit so ausdrucksvoll betonten Lauten und Silben, daß wir uns über sein Begehren nicht täuschen. Er erkennt sich und seine Umgebung, er ist sich seiner Stellung bewußt. [...] Seine Gefühle drückt er aus wie ein Mensch. In heiterer Stimmung lacht er freilich nicht, aber er schmunzelt doch wenigstens; trübe Stimmung dagegen bekundet er ganz in derselben Weise wie ein Mensch: man kann in seinem Gesichte lesen. (Brehm 1866, 231)

„Die Halbmenschlichkeit dieses Affen“ (Brehm 1866, 231) liegt für Brehm nicht zuletzt darin begründet, dass Molli sich fast wie ein Mensch verhalten und unterhalten kann. Das Bedeutungsspektrum der „Laute[ ] und Silben“ buchstabiert Brehm daher auch explizit aus, wenn er eine exemplarische Mensch-Tier-Interaktion zwischen Molli und sich selbst beschreibt:

\begin{abstract}
Molli sitzt ruhig in ihrem Käfig, betrachtet sich [sic] klugen Auges die sie anstarrenden Beschauer oder unterhält sich mit ihren Bekannten und Bekanntinnen [sic], als der Betreffende (meinetwegen ich selbst) eintritt, in der Absicht, sie zur Lustwandlung [...] abzuholen./ ,Molli, wollen wir spazieren gehen?`/ ,Oh!‘ antwortet sie freudig bejahend. [...] ,Gieb mir die Hand, Molli.`/ Sie thut es. ,Nein, die andere./ Sie wechselt./ ,Willst Du die Peitsche?`/ ,Oh, oh, oh!‘ - bedeutet: ,Ganz gewiß!` $(1866,232)$
\end{abstract}

Der „Pseudoanthropos“ (Brehm 1866, 231) weiß laut Brehm nicht nur genau, was er will, sondern auch, wie er diesen Willen kommunizieren kann. Für Brehm sind Mollis Laute sprachäquivalent, sie lassen sich dementsprechend auch problemlos in sprachliche Aussagen überführen: „Oh“ ist dann mal eine einsilbige Affirmation, mal kann es reduplizierend „Ganz gewiß“ heißen. Die Schimpansin versteht und kann verstanden werden.

Brehms Werk steht im Zeichen einer fundamentalen Emotionalisierung, Pädagogisierung und Moralisierung der Mensch-Tier-Beziehungen im 19. Jahrhundert (vgl. Eitler 2014). Tieren schrieb man nun nicht nur menschenähnliche Gefühle zu. Vielmehr forderte man gleichzeitig bestimmte (Mit-)Gefühle des Menschen für Tiere ein. ${ }^{6}$ Dass Tiere ihre Gefühle äußern und somit proto-sprachlich zum Ausdruck bringen können, wurde in diesem Zuge zum Gemeinplatz der Vor-

6 Vgl. zu diesen kultur- und gefühlsgeschichtlichen Wandlungsprozessen insbesondere Buchner-Fuhs 1996 und 1998. 
stellung von der ,Natur' der Tiere. Mit dem Sprechen über und für Molli präfiguriert Brehm ein gängiges poetologisches Modell literarischer Zoographien des 20. und 21. Jahrhunderts. Biographierung ist hier immer auch gleichbedeutend mit einem Sprechen über die Sprache der Tiere: Die Tierbiographie ist somit gleichzeitig auch ein Ort, an dem ein assimilationistisches Argument (Tiere haben einen sprachanalogen ,Selbstausdruck') stark gemacht wird. Die Sprache der Tiere in ihren Biographien möchte ich nun exemplarisch anhand von zwei Texten aus dem 20. und 21. Jahrhundert aufzeigen.

\section{Ornitho-Biographie}

Schon in Brehms Bericht aus dem Leben der Schimpansin Molli war die Relationalität von menschlicher und tierlicher ${ }^{7}$ Biographie augenscheinlich geworden. Brehm biographiert Tiere nicht nur, sondern setzt sich zugleich auch selbst autobiographisch in Szene. Und Brehm beschreibt die Schimpansin nicht einfach, sondern verschriftlicht seine Perspektive auf die Beziehung und Interaktion mit diesem Individuum, das er in der Tat wie eine Person behandelt und ausdeutet.

Die literarischen Tierbiographen des 20. und 21. Jahrhunderts haben diese Relation verinnerlicht. Auch hier entpuppt sich der biographische Tier-Text angesichts der Tatsache, dass die Lebensverläufe von menschlichem Biographen und tierlichem Protagonisten durch das gemeinsame Zusammenleben untrennbar miteinander verquickt sind, als durchweg autobiographischer. Wie bei Brehm ist diese ,verdeckte Selbstinszenierung“ des Biographen bzw. der Biographin auch immer Ausdruck einer komplexen emotionalen Mensch-Tier-Beziehung. Zeitgenössische Tierbiographien präsentieren sich dabei als Signum einer zunehmenden Sentimentalisierung, Familiarisierung und (marketingorientierten) Instrumentalisierung von Tieren. ${ }^{8}$ Tiere können hier als eigenwerbungswirksame Sprachrohre ihrer ,berühmten“ Besitzerinnen und Besitzer, ${ }^{9}$ vor allem

7 Im Folgenden verwende ich den Begriff ,tierlich“ in Analogie zu ,menschlich‘. Hiermit sei eine Distanzierung von den abwertenden Konnotationen angezeigt, die häufig mit dem Begriff ,tierisch` einhergehen.

8 Vgl. in diesem Zusammenhang Huff und Haefer 2012.

9 Besonders deutlich wird diese Tendenz in quasi-autobiographischen Ich-Romanen, die Tieren nicht nur prosopopoietisch eine Stimme geben, sondern diese Tiere auch von ihrem Zusammenleben mit ihren ,besonderen` Besitzerinnen und Besitzern erzählen lassen, vgl. z. B. Bush 1990, Glööckler 2013. 
aber als Freunde oder, wie es auch bei Verdaguer heißt, als „das beste Stück der Familie“ (Verdaguer 1966, 83) firmieren. Joaquin Verdaguer i Travessi (18981966), mallorquinischer Journalist und Schriftsteller, reflektiert diese emotionale Nähe und deren Bedeutung für seinen Schreibprozess in der Lebensgeschichte eines kleinen Vogels, in der er das dreizehnjährige Leben von und Zusammenleben mit Tito, einem mallorquinischen Kanarienvogel, erzählt, von Beginn an:

Ich schreibe diese Biographie nicht, von außen', wie es dem objektiven Biographen geziemt, der etwas auf sich hält, sondern von ,innen'. [...] Auch bemerke ich vor dem Beginn schon mit Schrecken, daß ich bereits bis an den Hals darin stecke. [...] Und dieser mein Fehler, diese meine Schwäche sind Schuld daran, daß jedes Wort, aus dem die Lebensgeschichte sich zusammensetzt, ein kleines Herz und ein Fünkchen meiner Seele trägt. (Verdaguer 1966, 10)

In das textuelle Geflecht der literarischen Zoographie, so gibt Verdaguer an dieser Stelle zu verstehen, hat sich der Biograph als emotional Beteiligter buchstäblich mit Leib und Seele eingewoben. Die Lebensgeschichte „der kleinen Vogelpersönlichkeit“ (Verdaguer 1966, 85) setzt hier mit einem auto(r)reflexiven Geständnis ein, das die Lebens- gewissermaßen als Liebesgeschichte und das Sprechen über ein Vogelleben als Erzählakt apostrophiert, der Menschen- und Vogelleben zusammendenkt.

Berichtet wird über Tito, so der offizielle Name des ,Findelkindes‘, das auch als „Brüderchen Vogel“ (Verdaguer 1966, 22), „unser Hätschelkind“ (25), als „Kamerad“ und „Freund“ (57) referenziert wird, in Analogie zu einem historiographisch-biographischen Erzählmodell: „Dem Beispiel aller Geschichtsschreiber getreu, "schreibt Verdaguer in einer Art Zwischenfazit,

habe ich Ihnen, lieber Leser, erzählt, wie Tito in unser Haus kam, wie er sich der Familie eingliederte, wie er unsere Herzen eroberte und wir das seine, wie nacheinander seine Kindheit, seine Jugend und sein Erwachsensein verliefen, welche Episoden Marksteine in seinem Leben bildeten - so der Beginn seiner herrlichen Befiederung, der Ausbruch seines ersten Gesanges und so weiter ... wie sein ganz zärtliches Leben, in engster Gemeinschaft mit dem von uns dreien, Tag um Tag dahinglitt. $(1966,63)$

Tito erhält unmittelbar nach seinem Einzug in Verdaguers Leben den Status eines Familienmitgliedes. Der Drei-Personen-Haushalt, der sich aus Verdaguer und seinen Schwestern zusammensetzt, hat Zuwachs bekommen: „Ich [...] faßte die Gefühle von uns dreien in die Worte zusammen: ,Nun sind wir also vier.““ (Verdaguer 1966, 18) Tito nimmt nicht nur an allen Mahlzeiten teil und küsst Verdaguer mit dem Schnabel ,beim Zubettbringen, beim Aufstehen, beim Abschiednehmen, beim Wiedersehen - und wer weiß, wie oft noch“ (Verdaguer 1966, 66), sondern 
ist laut Selbstauskunft des Autors auch konstitutiv an der Produktion des Textes beteiligt:

\begin{abstract}
Nicht nur auf dieser Seite, sondern auf jeder, die ich über ihn schreibe, ist mein kleiner Vogel gegenwärtig und gibt den Ton an. Aufmerksam, fröhlich und zärtlich verfolgt er den Lauf meiner Feder, pickt mir leicht in die Finger, als wolle er mir diktieren und schlägt mit den Flügeln voll Stolz über das, was wir miteinander verfassen. [...] Natürlich kann es da nicht ausbleiben, daß ich recht häufig ,auf Vogelart‘ schreibe, ,en oiseau', wie es so schön auf französisch heißt. $(1966,24)$
\end{abstract}

Verdaguer betrachtet die Lebensgeschichte eines kleinen Vogels - aller Selbstironie zum Trotz - als interspezifisches, zoopoetisches Kollaborativ-Projekt. ${ }^{10}$ Nicht nur habe sich der Biograph in die Geschichte seines Protagonisten eingeschrieben, in gewisser Weise habe auch der kleine Tito die Feder, wenn nicht geführt, so doch die federführende Instanz wesentlich mit seiner Präsenz und vor allem seiner Persönlichkeit beeinflusst. Tito wird im Zuge dieser autoreflexiven Geste der Status eines textkonstituierenden Akteurs zugewiesen.

Verdaguer begreift die Vogelbiographie als Mittel der Verlebendigung, vor allem aber Verewigung eines „kleinen Helden“ (1966, 53), einer „Kleinigkeit“ (10), die große Gefühle evozierte.

In diesem Zuge spricht Verdaguer allerdings nicht nur über Tito und seine (quasi-autobiographische) Ko-Autorschaft, sondern auch über die ,Sprache“ des Vogels und die Formen der Mensch-Vogel-Kommunikation. Bereits die erste Begegnung zwischen Biograph und biographischem Subjekt steht unter dem Vorzeichen eines interspezifischen Sprachkontaktes. Verdaguer ist der gerade einmal eine Woche alte Vogel in die Hand gelegt worden: „Ich lächelte einfältig, wiegte ihn ein wenig wie ein Kind, das man einschläfern will, hob ihn näher an mein Gesicht, um ihn besser zu sehen, und sagte zu ihm: ,Pi-pi-pi!‘ Ich kam mir vor wie eine Mutter.“ $(1966,8)$ Während Verdaguer das Vogelkind anthropomorphisiert, mimt er selbst eine zoomorphe Figur, die mit ,Vogellauten' einen Kontakt jenseits der haptischen Berührung herzustellen bestrebt ist.

Dass Vögel ein Selbstausdrucks- und Kommunikationsvermögen besitzen, wird im Verlauf des Textes mehrfach betont und ästhetisch konturiert. Für Verdaguer sind Vögel beredte Wesen, die „zur Verständigung miteinander keiner Sprache bedürfen.“ $(1966,78)$ Im „unmittelbarem Kontakt“ gebe Tito über sich auch gegenüber dem Menschen Auskunft, und zwar mit

10 Vgl. zu diesen Konzepten Herman 2016, Moe 2014. 
de[m] Ausdruck seiner Augen, de[m] Tonfall seiner Stimme, d[em] Tempo seiner Bewegungen, d[em] verschiedenartige[n] Zittern seiner Flügel, d[er] Haltung seines Kopfes, d[er] Stellung seines Schnabels, sein[em] Beben vor Freude oder Zorn, seine[r] Art zu reagieren, sein[em] Gesang. (Verdaguer 1966, 79)

Den Körper, das Verhalten und die Laute des Vogels präsentiert Verdaguer als semiotisches Zusammenspiel, das der Mensch im Sinne einer ,ornithologischen Semantik' am Leitfaden der Physis und des Klanges erschließen kann: Auch ohne eine sprachliche Rede weiß sich Tito daher, laut Verdaguer, „auf beredte Weise“ (1966, 79) mitzuteilen. Zwei Darstellungsverfahren untermalen diese These. Zum einen spricht Verdaguer mithilfe der Prosopopöie stellvertretend für den Vogel und ,übersetzt den nonverbalen Selbstausdruck des Vogels im Zuge einer interpretativen Projektion. Tito wird hier als sprachlose, ,natürliche ' Chiffre inszeniert, die sich gleich einem offenen Buch lesen und dekodieren lässt: „Aus seinen Augen, aus seiner Haltung lese ich deutlich, daß er zu mir sagt: ,Wie gut haben wir es miteinander! Wie glücklich sind wir doch, wir zwei.““(Verdaguer 1966, 37) Die Prosopopöie, die „konkrete Dinge oder abstrakte Begriffe und Abwesende als redende Personen auftreten [läßt]“ und ,ihnen eine Stimme [verleiht]“ (Menke 2000, 137), wird hier als rhetorische ,Übersetzungsfigur‘ verwendet, die Körper- und Lautsprache im Sinne einer Verhaltensdeutung ausbuchstabiert: „Mit halbgeöffnetem Schnabel sah er mich an, was bei Vögeln einen Wunsch ausdrückt. Er sah mich an: Verstehst du mich nicht?‘, schien er zu fragen“ (Verdaguer 1966, 51).

Zum anderen arbeitet Verdaguer mit onomatopoetischen Umschriften. Titos „gewohnte[r] Gruß der Wiedersehensfreude“ sei ein „liebenswürdiges ,cri-cricri““ (Verdaguer 1966, 53). Das „Alphabet eines Vogellebens“ (Verdaguer 1966, 9) setzt sich für Verdaguer aus wenigen reduplizierten Silben zusammen, aus ,,cricri-cri', ,uit-uit-uit', ,pi-pi, piii-tiu' und eine Unmenge anderer Variationen, die keineswegs Wörter, sondern etwas weit über alle Wörter hinaus waren.“ $(1966,79)$ Jeder dieser Laute erscheint für Verdaguer als ein Quasi-Analogon der menschlichen Sprache, das dementsprechend auch alphabetisch repräsentiert und in einem zweiten Schritt unter Rückgriff auf die Prosopopöie interpretativ ausbuchstabiert werden kann.

Mit der Lebensgeschichte eines kleinen Vogels spürt Verdaguer somit nicht nur einer Sprache nach, die sowohl ein „Vogelmikroleben[ ]“ als auch menschliche Gefühle für dieses Vogelleben abzubilden imstande ist und auf diese Weise „die überklugen Buchstaben einer solchen Kleinigkeit [d.i. Tito] wegen bemüh[t]“ (Verdaguer 1966, 10). Der Text arbeitet sich vielmehr auch daran ab, den nonverbalen Protagonisten als ,beredtes 'Wesen ansichtig und eine Kommunikation jenseits der Speziesgrenzen glaubwürdig werden zu lassen. Rhetorische Anthropomorphisierung und Personifikation stehen in einem poietischen Zusammenhang, 
der dem biographischen Subjekt Intentionalität, Personalität und Ausdrucksvermögen sowie ein bestimmtes Sprachverständnis zugesteht. So schreibt Verdaguer über seine interspezifische ,Gesprächsführung': „Ich spreche mit leiser Stimme $\mathrm{zu}$ ihm und genauso als spräche ich mit einem Menschen. Das ist die richtige Art mit seinen Vogelfreunden umzugehen. Und er versteht mich über das gesprochene Wort hinaus.“ (1966, 26-27) Mensch und Vogel können sich gemäß dieser Logik zu verstehen lernen. Aus Verdaguers Perspektive wird auf diese Weise eine Interaktion von Angesicht zu Angesicht, ein gegenseitiges Verständnis buchstäblich auf Augenhöhe möglich: „Ich schaue ihn an, und er schaut mich an. Glückseligkeit sprüht aus seinen Augen. Ich spreche mit ihm, und aufmerksam lauscht er meinen Worten. Sein leidenschaftliches kleines Herz kommt mir von Tag zu Tag näher.“ (Verdaguer 1966, 29) Mit Tito sprechen heißt hier immer auch, dem Vogel ein quasi-autobiographisches Vermögen zuzusprechen. Die Sprache der Vogelbiographie geht im Zeichen einer Zuschreibung kognitiver und emotionaler Eigenschaften auf, die im Kontext assimilationistischer Vorstellungen von den Gemeinsamkeiten zwischen Mensch und Tier zu verorten ist.

\section{Katzengeflüster}

Auch die homodiegetische Erzählinstanz von Dewey stellt sich explizit als Autorin des Textes vor (vgl. Myron 2010 [2008], 4). Ganz ähnlich wie Verdaguer lädt Vicki Myron die Erstbegegnung zwischen Mensch und Tier in der Retrospektive projektions- und prophetieartig mit Bedeutung auf: „When the kitten had looked into my eyes, [...] we had made a connection. He was more than just a cat to me. It had only been a day, but already I couldn't stand the thought of being without him.“ (Myron 2010 [2008], 16) Das emotionale Verhältnis zwischen Biographin und biographiertem Subjekt wird, auch darin Verdaguer ähnlich, von vornherein transparent gemacht:

I loved Dewey already [...]. We all loved Dewey. How could you resist his charm? He was beautiful, loving, social [...]. What I couldn't believe was how much Dewey loved us. How comfortable he seemed around strangers. His attitude seemed to be, how can anyone not love a cat? Or more simply, how can anyone resist me? Dewey didn't think of himself, I soon realized, as just another cat. He always thought of himself, correctly, as one of a kind. (Myron 2010 [2008], 21)

Myron konstatiert nicht nur, dass es Deweys einzigartiger Charakter und seine einzigartige Findelkind-Geschichte war, die den Kater weit über die Grenzen seines Zuhauses, einer Kleinstadtbücherei in Iowa, beliebt und buchstäblich erzählwür- 
dig gemacht haben. Vielmehr deutet Myron bereits an dieser Stelle imaginativprojektiv das Verhalten und Empfinden des Katers („Dewey loved us“; „His attitude seemed to be" ${ }^{11}$ und suggeriert, dass Dewey sich seiner Besonderheit und somit seiner selbst bewusst war. Bereits am Ende des zweiten Kapitels sind auf diese Weise sowohl Deweys Biographiewürdigkeit postuliert als auch das stellvertretende Sprechen für Dewey und seine ,attitude“ im Sinne einer Verhaltensdeutung als ästhetisches Repräsentationsverfahren des Textes zur Darstellung gebracht worden.

Deweys Geschichte zeichnet der Text in Form einer relationalen Biographie nach, die nicht nur von Deweys und Myrons, sondern auch von denjenigen Leben erzählt, die durch Dewey verändert wurden. Den Beginn der Lebensgeschichte markiert der Tag im Januar 1988, an dem ein ausgesetztes Katzenkind in der Bücher-Rückgabeklappe der bereits erwähnten Bücherei gefunden wird. In kurzer Zeit entwickelt sich der Kater vom Attraktor der Beleg- und Kundschaft der Bücherei schrittweise zum medialen Ereignis (vgl. Myron 2010 [2008], 177, 216-219):

Dewey's face, his personality, his story, it all magnified. He received letters from Taiwan, Holland, South Africa, Norway, Australia. He had pen pals in half a dozen countries. A ripple started in a little town in northwest Iowa, and somehow the human network carried it all over the world. (Myron 2010 [2008], 199)

Der Text setzt Dewey als eine Persönlichkeit in Szene, der Prominenz- und KultCharakter zugesprochen wird: „It wasn’t just a great story. Dewey had charisma, like Elvis or any of the other people who will live in our minds forever.“ (Myron 2010 [2008], 209) Der Vergleich mit einem Popstar ist für Myron schon deshalb nicht abwegig, weil der Tod des neunzehnjährigen Katers in den Fernsehnachrichten verlesen (vgl. 2010 [2008], 260-61) und Myrons Buch über Deweys Lebensgeschichte international über drei Millionen Mal verkauft wurde (vgl. 2010 [2008], 273).

Die Biographie des Katers fungiert für Myron dabei als eines der Medien, die den toten Kater sowie seine besondere Lebens- und Wirkungsgeschichte monumental verewigen sollen. Im Nachwort hält sie fest: „Dewey’s memory will live on, I feel confident of that. [...] Maybe at the library, where his portrait hangs beside the front door [...]. Maybe in the children who knew him [...]. Maybe in this book.

11 Vgl. dazu auch „The Daily Routine“ (Myron 2010 [2008], 155-156) sowie „Dewey’s Job Description“ (Myron 2010 [2008], 203), die sowohl den Lebensalltag des Katers als auch seine soziale Funktion im Mikrokosmos der Bücherei reflektieren. In den „Basic Rules for Cats Who Have a Library to Run (according to Dewey Readmore Books)“ (Myron 2010 [2008], 166-167) wird auch das projektiv-prosopopoietische Sprechen für Dewey als Textverfahren sichtbar. 
After all, that's why I’m writing it. For Dewey.“ (Myron 2010 [2008], 268). Erzählt wird auch hier nicht aus einer Position der Distanz, sondern aus einer Perspektive, die ein gemeinsames Leben rekonstruiert und vor allem ein imaginatives Einfühlen in das Erleben des Katers repräsentiert.

Als sprachbegabtes und intentional anthropomorph handelndes Individuum erscheint Dewey mithilfe von zwei Formen der Repräsentation: Erstens ist auch für Myron die Prosopopöie maßgeblich, um von und vor allem für Dewey zu sprechen. Prosopopoietisch werden das Verhalten des Katers, seine Körper- und Lautsprache im Text als selbstreferentielle Aussagen artikuliert. Besonders deutlich wird diese Verschränkung von Idiosynkrasie, Fürsprache und Komik im Hinblick auf die Erzählung von Deweys Vorliebe für das Verspeisen von Gummibändern. Trotz kollektiver Mühen, diese Bänder vor dem Kater in der gesamten Bücherei zu verbergen, macht Dewey immer wieder einen schmackhaften Fund. Myron erzählt: „I turned around and walked back to the cabinet. Sure enough, there was Dewey, sitting on a shelf at eye level, a huge rubber band hanging out of his mouth. You can't stop the Dew! I'm going to be feasting for a week." (Myron 2010 [2008], 43) Diese Marotte des Katers wird im Text immer wieder komik-evozierend aufgerufen. Deweys erste Begegnung mit Plastik-Weihnachtsbaum und Baumschmuck kam für Dewey laut Myron beinahe einer Offenbarung gleich:

I could see his nose sniffing ninety odors a minute and his mind racing. Could it be? All this time, could Mom have been hiding the world's largest, most spectacular, most delicious smelly rubber band? When we pulled the Christmas tree out of the box, I could almost see Dewey's jaw drop. It's not a rubber band, it's ... it's ... better. (2010 [2008], 110)

Anthropomorphisierung und prosopopoietisches Sprechen sind hier eng miteinander verzahnt. Einerseits wird der Kater emotionalisiert, mentalisiert und personalisiert, andererseits macht der Text auf formaler Ebene unmissverständlich deutlich, dass Deweys ,Selbstaussage“ Myrons ,Fremdaussage“ darstellt: Syntaktisch unterscheiden sich Erzähler- und Figurenrede kaum; auf morphologischsemantischer Ebene lässt insbesondere der Begriff „Mom“ erkennbar werden, dass dieses stellvertretende Sprechen einer anthropomorphisierenden Projektion entspricht. Gespiegelt wird diese ,Infantilisierung' des Katers an einer anderen Textstelle durch Myrons Bezugnahme auf Dewey als „my baby boy, my baby Dewey.“ (2010 [2008], 136)

Zweitens arbeitet Myron nicht wie Verdaguer mit onomatopoetischer Verschriftlichung des tierlichen Selbstausdruckes, sondern beschreibt das Verhalten des Katers projektiv und ,paraphrasierend' im Sinne eines Sprechaktes. Als Myron erfährt, dass sie an Brustkrebs erkrankt ist, erscheint Dewey in ihren Augen als einzig ,verständnisvolle‘ Instanz: 


\begin{abstract}
Nobody understood what I was going through for those two years; nobody that is, but Dewey. [...] Every morning since his first week at the library, Dewey had waited for me at the front door. [...] Then, on one of the worst mornings of that terrible two years, he started waving. Yes, waving. I stopped and looked at him. He stopped and looked at me, then started waving again. It happened the next morning, too. [...] For the rest of his life, as soon as Dewey saw my car pull into the parking lot, he started scratching his right paw on the front door. [...] He was sitting there very still and waving at me, as if welcoming me to the library and, at the same time, reminding me, he was there. Every morning, Dewey waving at me as I walked toward the library made me feel better. (Myron 2010 [2008], 192)
\end{abstract}

Myron geht davon aus, dass der Kater nicht nur ein Gespür dafür besitzt, was in ihr vorgeht, sondern dass er ihr dieses Verständnis jenseits eines sprachlichen Ausdrucks auch vermitteln kann. Anstatt das Verhalten des Katers als instinktgebundenes Reiz-Reaktionsschema zu rationalisieren, wird es hier projektiv-anthropomorph als ein kommunikativer Akt, als rituelle Begrüßungsgeste interpretiert. Gleichzeitig markiert der Modus des Als-ob (,as if“) an dieser Stelle, inwiefern diese Lesart des Katzenverhaltens als subjektive Deutung zu verstehen ist. Die Annäherung an das, was Deweys nonverbaler Ausdruck bedeutet, wird hier gleichzeitig mit einem Index der Uneindeutigkeit versehen, der ein Eingeständnis hinsichtlich des Projektionscharakters eines Sprechens über die Erfahrungen und Absichten eines Tieres impliziert.

\title{
4 Die Sprachen der Tiere und ihrer Biographien
}

Mit Blick auf die im 19. Jahrhundert publizierten, didaktisch orientierten zoologischen Kollektivbiographien und die literarischen Zoographien von Bingley, Grube und Brehm hat in Verdaguers und Myrons Texten eine deutliche Verschiebung stattgefunden. Die literarische Zoographie leitet sich hier aus der Einzigartigkeit, nicht aus der (Arten-)Repräsentativität eines tierlichen Individuums ab. Das Erzählen vom Leben des Tieres hat hier immer auch eine monumentalisierende Funktion. Zum einen soll die tierliche Biographie die besondere Beziehung zwischen Tier und Biographin bzw. Biograph zum Ausdruck bringen, zum anderen dient der Text als eine Form der literarischen Verewigung, die dem Vergessen des Tieres entgegenwirken soll.

Darüber hinaus popularisieren Verdaguers und Myrons Texte assimilationistische Auffassungen: Tiere besitzen ein menschenähnliches Seelenleben und sind in der Lage, ihre Empfindungen, Überzeugungen und Absichten speziesübergreifend mitzuteilen. Sowohl Verdaguer als auch Myron gehen davon aus, dass der nonverbale Selbstausdruck ihrer Protagonisten verhaltenstheoretisch 
ausgedeutet und sprachlich ausgedrückt werden kann. Die literarischen Repräsentationsverfahren, die in ihren Texten maßgeblich sind, um diese Annahmen zu untermauern - Prosopopöie, Onomatopoesie, Paraphrase - haben dabei zwei inhärent paradoxe Effekte. Erstens kommen beide Texte nicht umhin, ihren epistemischen Anthropozentrismus einzugestehen. Menschen ist es nicht möglich, „,ihre spezifische menschliche Perspektivität zu umgehen“ (Grimm et al. 2016, 92). Sobald über das Verhalten und die Verhaltensmotive von Tieren gesprochen bzw. geschrieben wird, unterliegt die Darstellung einer menschlichen Perspektive und repräsentationalen Mitteln, die tierliches Erleben nicht mimetisch abbilden können. Die Qualia, ,wie es ist‘, die Welt als Vogel oder Katze zu erleben und sich dieser Welt nonverbal mitzuteilen, können Menschen und literarische Texte nicht jenseits anthropoperspektivischer Apriori und sprachlicher Repräsentation leisten. Die Sprache der Tiere in ihren Biographien macht demnach auf die Unhintergehbarkeit eines epistemischen Anthropozentrismus und auf die Aporien eines sprachlichen Zugriffs auf das nichtsprachliche Erleben der Tiere aufmerksam. Zweitens verweisen die literarischen Zoographien auf den Zuschreibungscharakter jedes menschlichen Sprechens über und für Tiere. Texte wie die Lebensgeschichte eines kleinen Vogels und Dewey sind sprachliche Konstrukte, in denen wiederum projektiv-imaginativ eine Sprache der Tiere entworfen wird. Während zoologische Texte - seien sie differentialistisch oder assimilationistisch fundiert - das Leben und Erleben der Tiere vermeintlich positivistisch im Sinne ,harter Fakten“ beschreiben, verdeutlicht das Sprechen für Tiere und ihren Selbstausdruck in literarischen Zoographien den Zuschreibungs- und Inszenierungscharakter jedes Sprechens über Tiere, ihr Erleben und Empfinden. Die Sprache der Tiere in ihren Biographien stellt somit vor Augen, dass ein Erzählen über und für Tiere (und ihre Sprache) weder voraussetzungslos noch unvermittelt ist. Tiere als denkende und fühlende Wesen wahrzunehmen und darzustellen ist nicht selbstverständlich, sondern das Ergebnis komplexer Zuschreibungen, Projektionen und Repräsentationsverfahren, die diese Annahmen und Wahrnehmungen erzählbar machen. Indem literarische Zoographien über das Sprechen der Tiere im biographischen Text sprechen, können sie den Konstruktcharakter jedes menschlichen Sprechens über das Erleben von Tieren zur Anschauung bringen und gleichzeitig das sprachliche Gemacht-Sein jeder (Tier-)Biographie ins Bewusstsein rufen. 


\section{Literaturverzeichnis}

Brehm, Alfred Edmund. Illustrirtes Thierleben. Eine allgemeine Kunde des Thierreiches. Bd. 1. Hildburghausen: Bibliographisches Institut, 1864.

Brehm, Alfred Edmund. Illustrirtes Thierleben. Eine allgemeine Kunde des Thierreiches. Bd. 2. Hildburghausen: Bibliographisches Institut, 1865.

Brehm, Alfred Edmund. „Bilder aus dem Thiergarten. Nr. 7. Molli.“ Die Gartenlaube. Illustrirtes Familienblatt 15 (1866): 229-232.

Bingley, William. Animal biography, or, Authentic anecdotes of the lives, manners, and economy, of the animal creation, arranged according to the system of Linnaeus. In Three Volumes. Second Edition. London: Richard Phillips, ${ }^{2} 1804$.

Buffon, Georges-Louis Leclerc de: Herrn von Buffons allgemeine Naturgeschichte. Eine freye mit einigen Zusätzen vermehrte Übersetzung nach der neuesten französ. Außgabe von 1769, hg. und übersetzt von Friedrich Heinrich Martini. Bd. 1. Berlin: Joachim Pauli, 1771.

Bush, Barbara. Millie's Book as Dictated to Barbara Bush. New York: William Morrow, 1990.

Buchner-Fuhs, Jutta. „Das Tier als Freund. Überlegungen zur Gefühlsgeschichte im 19. Jahrhundert.“ Tiere und Menschen. Geschichte und Aktualität eines prekären Verhältnisses. Hg. Paul Münch. Paderborn: Schöningh, 1998. 275-294.

Buchner-Fuhs, Jutta. Kultur mit Tieren. Zur Formierung des bürgerlichen Tierverständnisses im 19. Jahrhundert. Münster: Waxmann, 1996.

Eitler, Pascal. „Tiere und Gefühle: Eine genealogische Perspektive auf das 19. und 20. Jahrhundert.“ Tiere und Geschichte: Konturen einer ,Animate History“. Hg. Gesine Krüger, Aline Steinbrecher und Clemens Wischermann. Stuttgart: Franz Steiner, 2014. 59-78.

Glööckler, Harald. Billy King. Mein Leben mit Harald Glööckler. Falkensee: Tierisch Verlegen, 2013.

Grimm, Herwig et al. „Tierethik.“ Tiere. Kulturwissenschaftliches Handbuch. Hg. Roland Borgards. Stuttgart: Metzler, 2016. 78-97.

Grube, August W.: Biographieen aus der Naturkunde, in ästhetischer Form und religiösem Sinne. Nebst einem Worte über die ästhetische Seite des naturkundlichen Unterrichts. Stuttgart: Steinkopf, ${ }^{2} 1851$.

Herman, David. „Animal Autobiography; Or, Narration beyond the Human.“ Humanities 82, 5.4 (2016): https://doi.org/10.3390/h5040082

Ingensiep, Hans Werner. „Tierseele und tierethische Argumentationen in der deutschen philosophischen Literatur des 18. Jahrhunderts." NTM International Journal of History \& Ethics of Natural Sciences, Technology \& Medicine 4.1 (1996): 103-118.

Huff, Cynthia und Joel Haefner. „His Master's Voice: Animalographies, Life Writing, and the Posthuman." Biography 35 (2012): 153-169.

Menke, Bettine. Prosopopoiia. Stimme und Text bei Brentano, Hoffmann, Kleist und Kafka. München: Fink, 2000.

Middelhoff, Frederike. Literarische Autozoographien - Figurationen des autobiographischen Tieres im langen 19. Jahrhundert. Stuttgart: Metzler, 2020.

Moe, Aaron M. Zoopoetics. Animals and the Making of Poetry. Lanham: Lexington, 2014.

Müller-Tamm, Jutta (Hg.). Verstandenes Lebensbild. Ästhetische Wissenschaft von Humboldt bis Vischer. Eine Anthologie. Münster: LIT, 2010. 
Myron, Vicki. Dewey. The Small-Town Library Cat Who Touched the World. [2008]. New York und Boston: Grand Central, 2010.

Schmideler, Sebastian. „August Wilhelm Grube (1816-1884).“ Kinder- und Jugendliteratur. Ein Lexikon. Hg. Kurt Franz et al. 42. Ergänzungslieferung. Meitingen: Corian, 2011. 1-20.

Schmideler, Sebastian. „,Schwein, Sage deine Geschichte her! Tierdarstellungen in der Kinderund Jugendliteratur des 18. und 19. Jahrhunderts." InterJuli - Internationale Kinder- und Jugendliteraturforschung 1 (2012): 43-65.

Schnicke, Falko. „Begriffsgeschichte: Biographie und verwandte Termini.“ Handbuch Biographie. Methoden, Traditionen, Theorien. Hg. Christian Klein. Stuttgart und Weimar: Metzler, 2009. 1-6.

Toepfer, Georg. Historisches Wörterbuch der Biologie: Geschichte und Theorie der biologischen Grundbegriffe. Bd. 2. Darmstadt: Wissenschaftliche Buchgesellschaft, 2011.

Verdaguer, Joaquin. Lebensgeschichte eines kleinen Vogels. Aus dem Spanischen von Anne Grete Thormann. München: Heimeran, 1966.

Wild, Markus: Anthropologische Differenz. Der Geist der Tiere in der frühen Neuzeit bei Montaigne, Descartes und Hume. Berlin und New York: De Gruyter, 2006.

Frederike Middelhoff studierte deutsche und englische Literatur- und Sprachwissenschaft an der JMU Würzburg und an der University of Exeter (UK). Ihre Monographie Literarische Autozoographien - Figurationen des autobiographischen Tieres im langen 19. Jahrhundert erschien Anfang 2020 im Metzler Verlag. Seit März 2020 ist sie W1-Professorin für Neuere Deutsche Literatur mit dem Schwerpunkt Romantikforschung an der Goethe-Universität Frankfurt. 